\title{
Nachweis mehrerer Nucleaseaktivitäten im Liquor cerebrospinalis
}

\author{
Von E. J. ZöLlner, R. K. ZahN \\ Pbysiologisch-Cbemisches Institut (Direktor: Prof. Dr. R. K. Zabn) \\ und \\ G. MeINIG \\ Neurocbirurgische Klinik (Direktor: Prof. Dr., Dr. b. c. K. Schürmann) der Jobannes-Gutenberg-Universität Mainz \\ (Eingegangen am 12. September/10. November 1972) \\ Das komplexe Gefüge der Desoxyribonuclease (EC 3.1.4.5, EC 3.1.4.6)-Aktivitäten des menschlichen Liquor cerebrospinalis wurde mit \\ der Mikro-Disc-Elektrophorese aufgelöst. Dabei lassen sich sicben Banden hinsichtlich ihrer charakteristischen Wanderungsgeschwin- \\ digkeit, optimalem Substrat und optimalen Inkubationsbedingungen unterscheiden. Als Substrate wurden Herings-DNA und Kalbs- \\ thymus-DNA verwendet. Native DNA wurde mit denaturierter verglichen. Als weitere Kriterien dienten die unterschiedlichen Enzym- \\ aktivitäten bei pH 5,0 in $0,1 \mathrm{~mol} / 1 \mathrm{Na}$-Acetat-Puffer oder $\mathrm{pH} 7,4$ in $0,1 \mathrm{~mol} / 1$ Tris-HCl-Puffer, unter Zusatz von $\mathrm{MgCl}_{2}, \mathrm{CaCl}_{2}, \mathrm{EDTA}$, \\ $\mathrm{Na}_{2} \mathrm{SO}_{4}, \mathrm{NaF}$ und Mercaptoäthanol in verschiedenen Kombinationen.
}

\section{Detection of several different nuclease activities in buman cerebrospinal fluid}

The distribution pattern of deoxyribonuclease (EC 3.1.4.5, EC 3.1.4.6)-activities in human cerebrospinal fluid was examined by microdisc-electrophoresis. Seven fractions, differing in their electrophoretic mobility, in the nature of their optimal substrate and in their optimal incubation conditions, were characterized. Herring sperm DNA and calf thymus DNA were compared as substrates; DNA in its native form was compared with the denatured state. For further characterization, the various enzyme activities were tested at $\mathrm{pH} 5.0$ in $0.1 \mathrm{~mol} / 1 \mathrm{Na}$-acetate buffer or at $\mathrm{pH} 7.4$ in $0.1 \mathrm{~mol} / \mathrm{l}$ Tris- $\mathrm{HCl}$ buffer in the presence of different combinations of $\mathrm{MgCl}_{2}, \mathrm{CaCl}_{2}, \mathrm{EDTA}$, $\mathrm{Na}_{2} \mathrm{SO}_{4}, \mathrm{NaF}$ and mercaptoethanol.

Bisher finden sich in der Literatur nur spärliche und dazu noch divergierende Befunde über Desoxyribonucleasen im Liquor cerebrospinalis. Neben Ribonucleasen (EC 2.7.7.16) wurden bisher Desoxyribonucleasen (EC 3.1.4.5) (1) und unspezifische DNA spaltende Phosphodiesterasen (EC 3.1.4.1) (2) im Liquor cerebrospinalis nachgewiesen.

Jedoch konnte Kovacs (1) weder mit dem von ihm benutzten viskosimetrischen Desoxyribonuclase-Nachweis, noch mit der Messung säurelöslicher DNASpalt-Produkte, oder der Bestimmung der Extinktionszunahme bei $260 \mathrm{~nm}$ bei Normalpersonen Aktivitäten nachweisen. Dagegen fand er bei Probanden, die an Meningitis, Syphilis, Epilepsie, Hydrocephalus, Contusionen sowie einigen neurologischen oder neuropsychiatrischen Leiden erkrankt waren, Desoxyribonuclease-Aktivitäten. Die besonders starke Zunahme an Desoxyribonucleasen bei Meningitis erklärte er entweder mit einer verstärkten Durchlässigkeit der BlutHirn-Schranke oder als Folge mikrobieller Enzymabgabe.

HEALY (2) wies mit einem von ihm entwickelten immunologischen Phosphodiesterasenachweis sowohl bei gesunden Kontrollpersonen als auch bei neurologisch Kranken Aktivitäten nach. Dabei fand er starke Unterschiede, wenn et native mit denaturierter DNA als Substrat verglich. Dies erklärte er mit unterschiedlichen Aktivitäten an einer zusätzlichen, möglicherweise dem Serum entstammenden Desoxyribonuclease.
Unser Ziel ist es, Desoxyribonuclease-Aktivitäten im Liquor cerebrospinalis zu trennen und sie darüberhinaus aufgrund ihrer unterschiedlichen Eigenschaften im Mikromaßstab zu charakterisieren. Die von uns ge. wählte mikro-disc-elektrophoretische Methodik bietet mit ihrer hohen Empfindlichkeit und den guten Trenneffekten hierzu günstige Voraussetzungen. So läßt sich nicht nur der qualitative Nachweis unterschiedlicher Nucleaseaktivitäten führen, sondern es könnte vielleicht als weiterer Schritt die Zuordnung des Desoxyribonuclease-Musters zu einzelnen neurologischen Krankheitsbildern möglich werden.

\section{Material und Methoden}

Als Untersuchungsmaterial diente durch Lumbalpunktion gewonnener Liquor cerebrospinalis. Der Liquor cerebrospinalis wurde entweder baldigst, nachdem er höchstens $1 \mathrm{~h}$ bei $4^{\circ} \mathrm{C}$ gestanden hatte, untersucht oder er wurde unmittelbar nach Entnahme auf mindestens $-20^{\circ} \mathrm{C}$ tiefgefroren. Dabei wurde nur nicht-haemorrhagischer, nicht xanthochromer Liquor cerebrospinalis verwendet.

Das Untersuchungsmaterial stammte von Patienten mit den Diagnosen:

Hypoglossus-Neurinom;

Myelopathie mit beginnender Paraparese beider Beine im Gefolge einer Spondylolisthesis tuberculosa mit 2 Jahren;

Nucleus-pulposus-Prolaps;

Mischung aus Liquores von Patienten mit Nucleus-pulposusProlaps und Melanoblastom des Auges.

Die jeweiligen Proben wurden mit Polyäthylenglykol oder mit Membranfiltern (Schleicher und Schüll) auf $1 / 10$ des Volumens eingeengt. Die Konzentrierung wurde bei $4^{\circ} \mathrm{C}$ und mit $5 \mathrm{mmol} / \mathrm{l}$ $\mathrm{CaCl}_{2}$ Lösung zur Stabilisicrung der Desoxyribonucleasen gegen 
Proteasen und gegen Inaktivierung durch Reduktion $(3,4)$ durchgeführt. Sie dauerte bei $1 \mathrm{ml}$ Ausgangsvolumen etwa $2 \mathrm{~h}$. Meßbare, über die Genauigkeit der Bestimmungsmethode hinausgehende Aktivitätsverluste waren dabei nicht feststellbar. Die Auftrennung der Liquor-Desoxyribonucleasen mit Hilfe der Míkro-DiscElektrophorese wurde im wesentlichen analog der Urin-Desoxyribonucleasen-Trennung (5) durchgeführt. Die Gele enthielten $134 \mathrm{~g} / 1$ Acrylamid und 0,3 g/l DNA. Dabei wandern die Desoxyribonucleasen nach Maßgabe ihrer elektrophoretischen Wanderungsgeschwindigkeit unterschiedlich weit in das DNA-haltige Trenngel unter Bedingungen ein, bei denen sie nicht aktiv sind. Nach Beendigung der Trennung wurden die Polyacrylamidgele in einen Inkubationspuffer überführt. Sind die Desoxyribonucleasen unter diesen Bedingungen aktiv, so entstehen DNABruchstücke, die aus dem Gel diffundieren. Nach Anfärbung der DNA-Gele mit Gallocyanin und nachfolgender Differenzierung, finden sich proportional der Desoxyribonuclease-Aktivität Zonen, die infolge ihres DNA-Verlustes weniger oder überhaupt nicht angefärbt sind.

Die Trennung erfolgte bei $700 \mu \mathrm{A}$ pro Gel und dauerte $100 \mathrm{~min}$ bei etwa $4^{\circ} \mathrm{C} .1 \mathrm{~g} / \mathrm{l} \mathrm{DNA}$ wurde im Trenngelpuffer $10 \mathrm{~min}$ auf $100^{\circ} \mathrm{C}$ erhitzt, in Eis abgekühlt und so denaturiert. Entweder wurde eine aus Heringssperma nach der Hitze-Detergens-Methode präparierte DNA (6) oder eine Kalbsthymus-DNA (Miles-Seravac) verwendet.

Nach der elektrophoretischen Trennung wurden die Gele entweder in $0,1 \mathrm{~mol} / \mathrm{l} \mathrm{Na}$-Acetatpuffer $(\mathrm{pH} 5,0)$ oder in $0,1 \mathrm{~mol} / 1$ Tris-HCl-Puffer ( $\mathrm{pH} 7,4)$, bei $37^{\circ} \mathrm{C}$ unter Zusatz von einer der folgenden Lösungen bei der angegebenen Endkonzentration $5 \mathrm{~h}$ lang inkubiert:

1. $0,01 \mathrm{~mol} / 1 \mathrm{MgCl}_{2}+1 \mathrm{mmol} / 1 \mathrm{CaCl}_{2} ;$, '

2. $0,01 \mathrm{~mol} / 1 \mathrm{MgCl}_{2}+1 \mathrm{mmol} / 1 \mathrm{CaCl}_{2}+0,05 \mathrm{~mol} / 1 \mathrm{EDTA}$;

3. $5 \mathrm{mmol} / 1 \mathrm{EDTA}$;

4. $0,01 \mathrm{~mol} / 1 \mathrm{Na}_{2} \mathrm{SO}_{4}$;

5. $0,01 \mathrm{~mol} / 1 \mathrm{NaF}$;

6. $0,01 \mathrm{~mol} / 1$ Mercaptoäthanol.

Danach wurden die Gele wie früher beschrieben (5) entwickelt und bezüglich ihrer Desoxyribonuclease-Aktivität densitometrisch vermessen.

Alle Banden wurden mit gleichbehandelten bekannten Konzentrationsteihen an Desoxyribonuclease I (Worthington) normiert.
Abb. 1

Densitometerkurven von Miliro-Disc-ElektrophoreseGelen zum Desoxyribonuclease-Nachweis. Laufzeit $100 \mathrm{~min}$ bei $700 \mu \mathrm{A}$ pro Gel.

$a_{1}$ ) Probe Liquor bei Hypoglossus-Neurinom: Inkubation 0,1 mol/1 Na-acetat-Puffer

a.) Probe Liquor $+5 \mathrm{mmol} / 1$ EDTA; kubation 0,1 mol/1 Tris-HCl-Puffer $\mathrm{pH} 7,4+$
$0,01 \mathrm{~mol} / \mathrm{l} \mathrm{MgCl}+1 \mathrm{mmol} / 1 \mathrm{CaCl}_{2} ;$

$\left.b_{1}\right)$ Probe Liquor bei Myelopathie: Inkubation $0,1 \mathrm{~mol} / \mathrm{l}$ Na-Acetat-Puffer pH 5,0 + 5 mmol/l EDTA;

$\mathrm{b}_{2}$ ) Probe Liquor bei Myelopathie: Inkubation

$0,1 \mathrm{~mol} / 1$ Tris-HCI-Puffer pH 7,4 + 0,01 mol/1

$\mathrm{MgCl}_{2}+1 \mathrm{mmol} / 1 \mathrm{CaCl}_{2}$;

$c_{1}$ ) Probe Liquor bei Nucleus-pulposus-Prolaps: Inkubation 0,1 mol/1 Na-Acetat-Puffer pH 5,0+ $5 \mathrm{mmol} / 1$ EDTA;

$c_{2}$ ) Probe Liquor bei Nucleus-pulposus-Prolaps: Inkubation $0,1 \mathrm{~mol} / 1$ Tris- $\mathrm{HCl}$-Puffer $\mathrm{pH} 7,4+$ $0,01 \mathrm{~mol}_{1} \mathrm{MgCl}_{2}+.1 \mathrm{mmol}_{1} \mathrm{CaCl}_{2}$
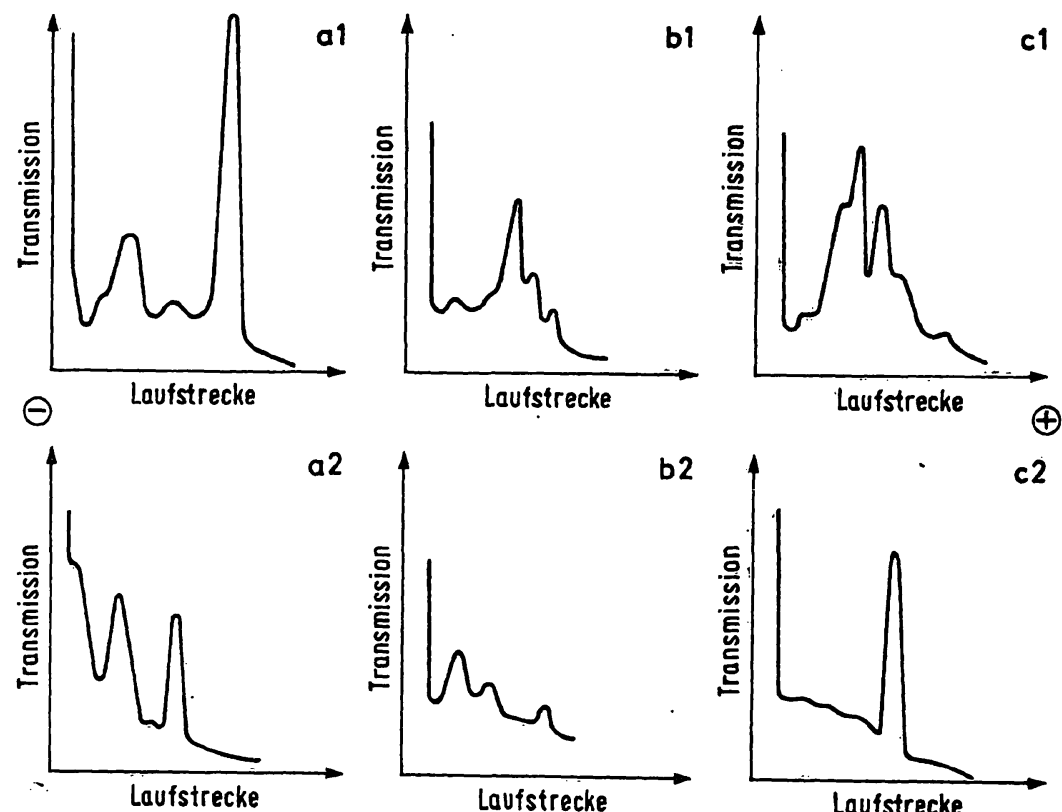

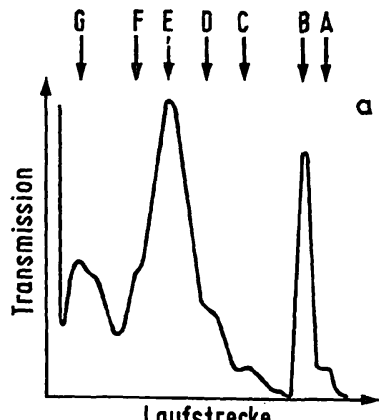

$\ominus$

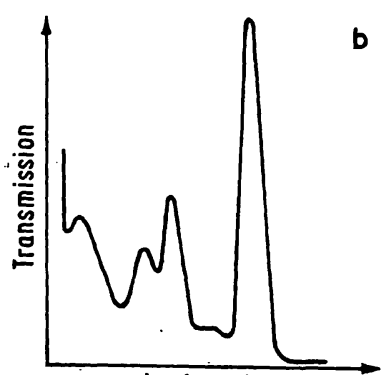

Laufstrecke

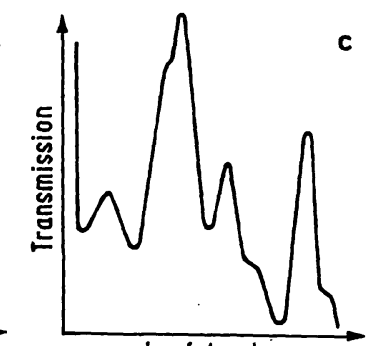

Loufstrecke

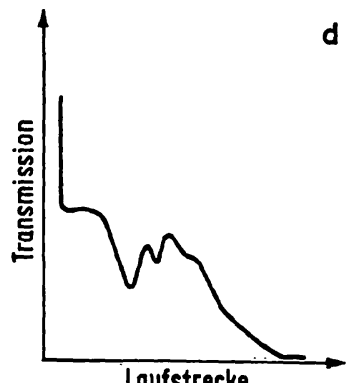

Laufstrecke

$d$
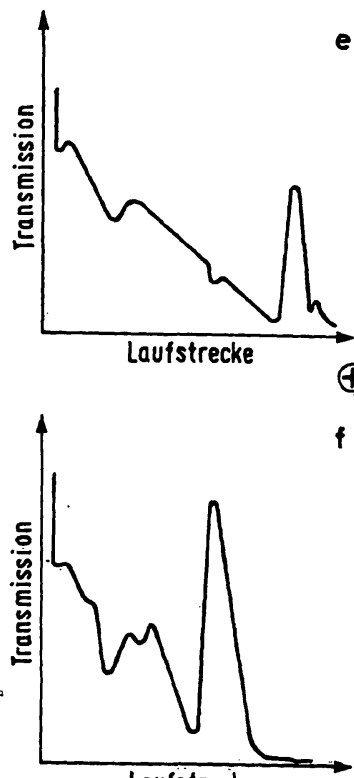

Abb. 2

Densitometerkurven von Mikro-Disc-Elektrophorese-Gelen zum Desoxyribonuclease-NachProbe Liquorgemisch Inkubation

a) $0,1 \mathrm{~mol} / 1 \mathrm{Na}$-Acetat-Puffer pH $5,0+0,01 \mathrm{~mol} / 1$ $\mathrm{MgCl}_{2}+1 \mathrm{mmol} / \mathrm{l} \mathrm{CaCl} ;$
b) $0,1 \mathrm{~mol} / 1 \mathrm{Tris}-\mathrm{HCl}$-Puffer $\mathrm{pH} \mathrm{7,4}+0,01 \mathrm{~mol} / 1$ c) $0,1 \mathrm{~mol} / 1 \mathrm{Na}-A$ cetat-Puffer $\mathrm{pH} 5,0+0,01 \mathrm{~mol} / 1$ c) d) 01 mol/ 1 Trisd) 1 mol/1 Tris-HCl-Puffer pH 7,4 + 0,01 mol/ e) $0,1 \mathrm{~mol} / \mathrm{l} \mathrm{Na}$-Acet $\mathrm{CaCl}_{2}+0,05 \mathrm{~mol} / 1 \mathrm{EDTA}$ $\mathrm{MgCl}_{2}+1 \mathrm{mmol} / \mathrm{CaCl}+0,01 \mathrm{~mol} / \mathrm{Na} \mathrm{mol} /$ $\mathrm{MgCl}_{2}+1 \mathrm{mmol} / \mathrm{C} \mathrm{CaCl}_{2}+0,01 \mathrm{~mol} / 1 \mathrm{Na}_{2} \mathrm{SO}_{4}$ $\mathrm{MgCl}_{2}+1 \mathrm{mmol} / \mathrm{CaCl}_{2}+0,01 \mathrm{~mol} / 1 \mathrm{Na} \mathrm{SO}_{4}$ 
Die Abhängigkeit det einzelnen Aktivitätsbanden von Inkubationsbedingungen und Substratangebot wurden mit einer Liquormischung untersucht. In dieser Mischung waren alle Banden gleichzeitig nachweisbar.

\section{Ergebnisse und Diskussion}

Die einzelnen Liquorchargen unterschieden sich hinsichtlich ihrer Nucleaseaktivitäten sowohl in der Anzahl der nachweisbaren Fraktionen, als auch in der Größe der Aktivitäten der nachweisbaren Banden voneinander (Abb. 1).

Sieben nach der Schnelligkeit ihrer anionischen Wanderungsgeschwindigkeit mit A-G bezeichnete DNAspaltende Aktivitäten konnten im Liquorgemisch isoliert dargestellt werden (Abb. 2).

Zur Charakterisierung der einzelnen Banden wurden sowohl die Inkubationsbedingungen als auch die angebotenen Substrate variiert. Die jeweiligen Aktivitäten konnten auch unter unterschiedlichen Bedingungen miteinander vergleichbar gemacht werden durch Planimetrieren der Densitometer-Ausschläge und Vergleich mit bekannten Desoxyribonuclease I- (Worthington) Konzentrationen (5).

Solche Ergebnisse sind in Tabelle 1, einige charakteristische Densitometerkurven in Abbildung 2 wiedergegeben.

Daraus läßt sich ersehen, daß drei Banden A, B und D ein klares Aktivitätsoptimum bei $\mathrm{pH} 5,0$ haben, die Banden $C$ und $G$ dagegen bei $p H$ 7,4. Zwei, die Banden $\mathrm{E}$ und $\mathrm{F}$, weisen bei beiden Inkubationsbedingungen deutliche Aktivitäten auf, die jedoch bei $\mathrm{pH}$ 7,4 um eine Zehner-Potenz absinken. Charakteristisch ist noch die Hemmbarkeit der Banden $C$ und $G$ durch EDTA, das die anderen Fraktionen unbeeinfluß täßt. Die Banden $E$ und $F$ sind in Gegenwart von Sulfat trotz sonst optimalen Inkubationsbedingungen kaum noch aktiv, A, B und D sind deutlich gehemmt.

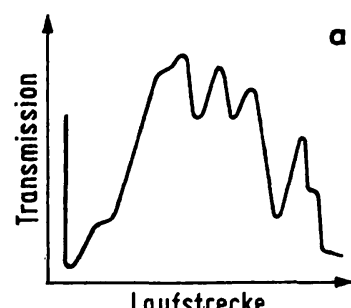

$\Theta$
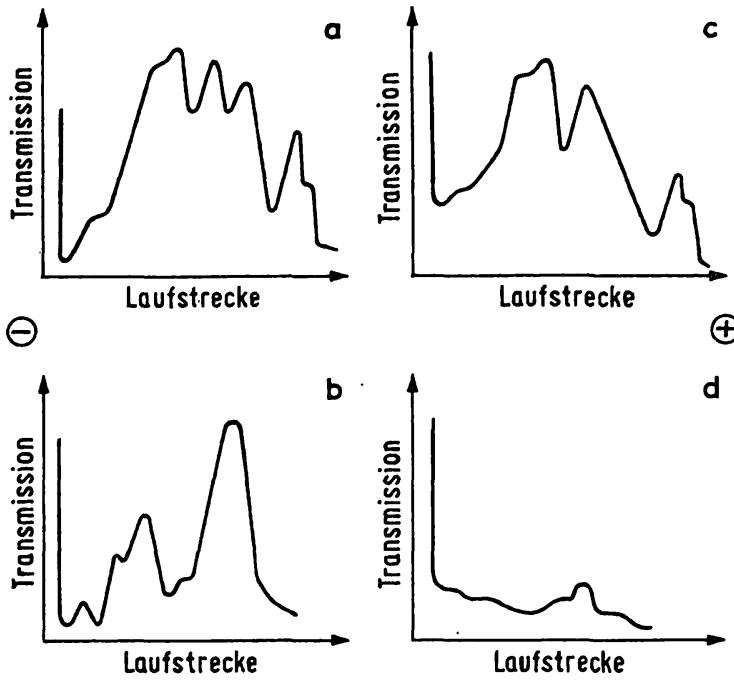

Abb. 3

Densitometerkurven von Mikro-Disc-Elektrophorese-Gelen zum Desoxyribonuclease-Nachweis. Laufzeit $100 \mathrm{~min}$ bei $700 \mu \mathrm{A}$ pro Gel Probe Liquorgemisch

a). native Kalbsthymus-DNA als Substrat; Inkubation in $0,1 \mathrm{~mol} / 1 \mathrm{Na}-$ Acetat-Puffer $\mathrm{pH} 5,0+5 \mathrm{mmol} / 1$ EDTA;

b) native Kalbsthymus-DNA als Substrat; Inkubation in $0,1 \mathrm{~mol} / \mathrm{l}$

Tris- $\mathrm{HCl}$-Puffer $\mathrm{pH} 7,4+0,01 \mathrm{~mol} / 1 \mathrm{MgCl}_{2}+1 \mathrm{mmol} / \mathrm{CaCl} \mathrm{Ca}_{2}$

c) denaturierte Kalbsthymus-DNA als Substrat; Inkubation in 0,1 mol/1

Na-Acetat-Puffer pH $5,0+5 \mathrm{mmol} / \mathrm{I}$ EDTA;

d) denaturierte Kalbsthymus-DNA als Substrat; Inkubation in $0,1 \mathrm{~mol} / \mathrm{l}$ Tris-HCl-Puffer $\mathrm{pH} \cdot 7,4+0,01 \mathrm{~mol} / \mathrm{l} \quad \mathrm{MgCl}_{2}+1 \mathrm{mmol} / 1$

Wird denaturierte DNA als Substrat angeboten (Abb. 3, Tab. 1), so verlieren im Vergleich zu nativer DNA C und $G$ stark, $E$ und $F$ deutlich an Aktivität, während A, B und D praktisch konstant bleiben. Der Austausch der Herings-DNA gegen Kalbsthymus-DNA (Abb. 3) erzeugt eine stärkere Aktivität der Fraktionen $C$ und D.

Die vorliegenden Befunde zeigen klar, daß im Liquor cerebrospinalis ein Gemisch verschiedener NucleaseAktivitäten vorliegt, die sowohl hinsichtlich ihrer Wanderungsgeschwindigkeit in der Polyacrylamid-Elek-

Tab. 1

Absolutwerte der einzelnen Banden unter verschiedenen Reaktionsbedingungen in Äquivalenten an Desoxyribonuclease I (g). Probe: Liquorgemisch. Zur Beurteilung der Desoxyribonuclease-Aktivitäten der einzelnen Banden wurden die Densitometerkurven bekannter Konzentrationen von Desoxyribonuclease 1 (Worthington, Freehold, USA, spez. Aktivität: 2500 Einheiten/mg) herangezogen. Als Bezugseinheit dient ein Äquivalent von $1 \mathrm{pg}$ Desoxyribonuclease I. Die planimetrierten Ausschläge der Densitometerkurven der Liquor-Trennungen wurden auf diese Eichlösungen bezogen

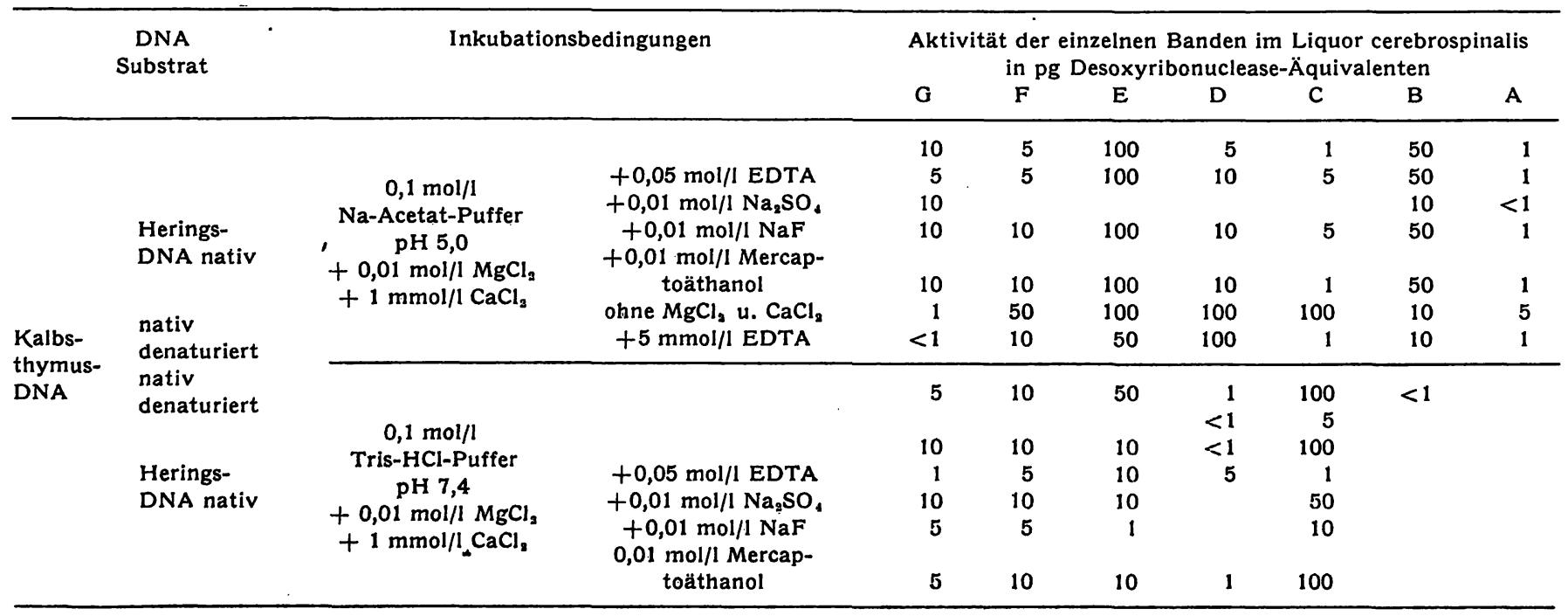


Tab. 2

Charakterisierung der einzelnen mittels Mikro-Disc-Elektrophorese in Liquor cerebrospinalis nachweisbaren Banden nach Substratspezifität, optimalen Inkubationsbedingungen und Hemmstoffen

\begin{tabular}{|c|c|c|c|c|}
\hline Bande & Optimales Substrat & Optimale Inkubation & $\begin{array}{l}\text { Gehemmt } \\
\text { durch }\end{array}$ & \\
\hline A & $\begin{array}{c}\text { Kalbsthymus }=\text { Herings-DNA } \\
\text { nativ }=\text { denaturiert }\end{array}$ & $\begin{array}{c}0,1 \mathrm{~mol} / 1 \mathrm{Na} \text {-acetat } \mathrm{pH} \\
5 \mathrm{mmol} / 1 \mathrm{EDTA}\end{array}$ & & \\
\hline B & Kalbsthymus $=$ Herings-DNA & $0,1 \mathrm{~mol} / 1 \mathrm{Na}$-acetat pH 5,0 & $\left(\mathrm{SO}_{4}^{2-}\right)$ & \\
\hline & nativ $=$ denaturiert & $5 \mathrm{mmol} / 1 \mathrm{EDTA}$ & & \\
\hline c & $\begin{array}{c}\text { Kalbsthymus > Herings-DNA } \\
\text { nativ } \gg \text { denaturiert }\end{array}$ & $\begin{array}{c}0,1 \mathrm{~mol} / \mathrm{l} \mathrm{Tris}-\mathrm{HCl} \mathrm{pH} 7,4 \\
0,01 \mathrm{~mol} / 1 \mathrm{MgCl}_{2}, 1 \mathrm{mmol} / 1 \mathrm{CaCl}_{2}\end{array}$ & EDTA & \\
\hline $\mathrm{D}$ & $\begin{array}{c}\text { Kalbsthymus }>\text { Herings-DNA } \\
\text { nativ }=\text { denaturiert }\end{array}$ & $\begin{array}{c}0,1 \mathrm{~mol} / 1 \mathrm{Na} \text {-acetat pH } 5,0 \\
5 \mathrm{mmol} / \mathrm{l} \text { EDTA }\end{array}$ & $\mathrm{SO}_{4}^{2-}$ & \\
\hline $\mathrm{E}$ & $\begin{array}{c}\text { Kalbsthymus }=\text { Herings-DNA } \\
\text { nativ }>\text { denaturiert }\end{array}$ & $\begin{array}{c}0,1 \mathrm{~mol} / 1 \mathrm{Na} \text {-acetat pH } 5,0 \\
5 \mathrm{mmol} / 1 \mathrm{l} \text { EDTA }\end{array}$ & $\mathrm{SO}_{4}^{2-}$ & \\
\hline $\mathrm{F}$ & $\begin{array}{c}\text { Kalbsthymus }=\text { Herings-DNA } \\
\text { nativ }>\text { denaturiert }\end{array}$ & $\begin{array}{c}0,1 \mathrm{~mol} / \mathrm{l} \mathrm{Na}-\text { acetat pH } 5,0 \\
5 \mathrm{mmol} / \mathrm{l} \text { EDTA }\end{array}$ & $\mathrm{SO}_{4}^{2-}$ & - \\
\hline G & $\begin{array}{c}\text { Kalbsthymus }=\text { Herings-DNA } \\
\text { nativ } \gg \text { denaturiert }\end{array}$ & $\begin{array}{c}0,1 \mathrm{~mol} / \mathrm{l} \mathrm{Tris}-\mathrm{HCl} \mathrm{pH} \mathrm{7,4} \\
0,01 \mathrm{~mol} / 1 \mathrm{MgCl}_{2}, 1 \mathrm{mmol} / 1 \mathrm{CaCl}_{2}\end{array}$ & EDTA & \\
\hline
\end{tabular}

trophorese als auch ihrer optimalen Inkubationsbedingungen zu unterscheiden sind (Tab. 2). Dabei ist ein Aktivitätsoptimum bei gleichen Reaktionsbedingungen als alleiniges Kriterium für eine gegebene Fraktion kein für eine Charaliterisierung ausreichendes Merkmal. Dies bedeutet, daß Untersuchungen über Desoxyribonucleasen im Liquor cerebrospinalis das aufgetrennte Aktivitätsspektrum berücksichtigen müssen, da andernfalls die Konzentrationserhöhung einer Fraktion durch Verminderung einer anderen überdeckt werden kann.

Das so erhaltene Spektrum an DNA-spaltenden Aktivitäten im Liquor cerebrospinalis soll als Grundlage dienen, um in weiteren Untersuchungen zu klären, ob die Zunahme an Desoxyribonuclease-Aktivität im Liquor cerebrospinalis bei einigen Erkrankungen (1) auf die Aktivitätszunahme einzelner Fraktionen zu- rückzuführen ist. Die Abklärung dieser Frage kann sowohl diagnostische Konsequenzen haben, als auch Einblick in die biologische Rolle dieser einzelnen Fraktionen erlauben. Eine Zuordnung der einzelnen Aktivitäten zu bekannten Säugetier-Désoxyribonucleasen $(7,8)$ ist aufgrund der bisher erhaltenen Kriterien noch nicht möglich, da pH-Optimum und Ionenabhängigkeit allein nicht ausreichen. Dies ist weiteren Untersuchungen über die Natur der durch ihren Angriff entstandenen DNA-Bruchștücke und über spezifische Proteininhibitoren vorbehalten.

\section{Danksagung}

Herrn Prof. Dr. S. Wende (Institut für Neuroradiologie) danken wir für die Überlassung von Untersuchungsmaterial, der Fa. H. MACK Illertissen für die Überlassung hochpolymerer DNA. Teile der Untersuchungen wurden durch eine Leibgabe der Deutschen Forschungsgemeinschaft unterstützt.

\section{Literatur}

1. Kovacs, E. (1954), J. Pediatr. 45, 569-574. - 2. Healy, J. W. (1964), J. Neurochem. 11, 119-123. - 3. Price, P. A., Lin, T.-Y., Stern, W. H. \& Moore, St. (1969), J. Biol. Chem. 244, 917-923. - 4. Price, P. A., Stein, W. H. \& Moore, St. (1969), J. Biol. Chem. 244, 929-932. - 5. ZöLLner, E. J., Müller, W. E. G., Zahn, R. K., Brehm, G. \& Korting, G. W.
(1971), Klin. Wochenschr. 49, 1290-1294. - 6. ZAFN, R. K., Tiesler, E., Kleinschmid, A. K. \& Lang, D. (1962), Biochem. Z. 336, 281-298. - 7. LAskowskr, M. SR. (1971), in The Enzymes (Boyer, PAUL D. ed.) 3rd edition Vol. 4, p. 289-311, N. Y. Acad. Press. - 8. Bernardi, G. (1971), in The Enzymes (Boyer, Paul D. ed.) 3rd edition Vol. 4, p. 271-287, N. Y. Acad. Press.

\section{Dr. E. Jürgen Zöllner}

Physiologisch-Chemisches Institut der Johannes-Gutenberg-Úniversität 65 Mainz

Johann-Joachim-Becher-Weg 13 